\title{
The expression of DBC1/CCAR2 is associated with poor prognosis of ovarian carcinoma
}

\author{
Dong Hyu Cho', Ho Sung Park², See-Hyoung Park ${ }^{3}$ Kyoung Min Kim², Myoung Ja Chung ${ }^{2}$, Woo Sung Moon², \\ Myoung Jae Kang ${ }^{2}$ and Kyu Yun Jang ${ }^{2^{*}}$
}

\begin{abstract}
Background: Recent reports have shown that deleted in breast cancer 1 (DBC1/CCAR2) is an indicator of poor prognosis of various human cancers. However, its expression in ovarian carcinoma has not been reported.

Methods: We investigated the immunohistochemical expression of DBC1 and BRCA1 and their prognostic significance in 104 ovarian carcinomas. Survival analyses were performed according to the Kaplan-Meier method, as well as univariate and multivariate Cox proportional hazard regression analysis.

Results: Positive expression of DBC1 and BRCA1 were seen in 63\% (66/104) and 44\% (46/104) of overall ovarian carcinomas, respectively. DBC1 expression was significantly associated with advanced clinicopathological factors such as high tumor stage, latent distant metastasis, platinum-resistance, elevated serum levels of CA125, high histologic grade, and BRCA1 expression. In the histological subtypes of ovarian carcinomas, DBC1 expression was more common in serous carcinoma $(72 \%, 54 / 75)$ than mucinous carcinoma $(15 \%, 3 / 20)$. BRCA1 expression was significantly associated with latent distant metastasis, platinum-resistance, and higher histologic grade. In addition, DBC1 expression was significantly associated with shorter overall survival (OS) and relapse-free survival (RFS) in 104 ovarian carcinomas (OS; $P<0.001, \mathrm{RFS} ; P<0.001$ ) and 63 high-grade serous carcinomas (OS; $P=0.008, \mathrm{RFS} ; P=0.023$ ) by univariate analysis. BRCA1 expression was significantly associated with OS and RFS in 104 ovarian carcinomas (OS; $P=0.005, R F S ; P=0.002$ ) and 75 serous carcinomas (OS; $P=0.047, \mathrm{RFS} ; P=0.038$ ) by univariate analysis. Moreover, $\mathrm{DBC} 1$ expression was an independent prognostic indicator for OS in both 104 ovarian carcinomas $(P=0.021)$ and 63 high-grade serous carcinomas $(P=0.011)$ by multivariate analysis.

Conclusions: These results indicate that the expression of DBC1 and BRCA1 are closely related with in the progression of ovarian carcinomas and may have clinical utility in the prediction of prognosis of ovarian carcinomas. Especially, DBC1 expression could be employed as a significant prognostic indicator for ovarian carcinomas especially in high-grade serous carcinomas.
\end{abstract}

Keywords: Ovarian neoplasms, Serous carcinoma, Deleted in breast cancer 1, BRCA1, Prognosis

\section{Background}

Deleted in breast cancer $1 /$ cell cycle and apoptosis regulator 2 (DBC1/CCAR2) was named by its deletion at a region 8 p22 in breast cancer. Thereafter, $\mathrm{DBC} 1$ was redesignated CCAR2 to eliminate possible confusion with deleted in bladder cancer 1 and because it has partial sequence homology to CCAR1. The deletion of DBC1 in breast cancer suggested it may have a role as a tumor

\footnotetext{
*Correspondence: kyjang@chonbuk.ac.kr

${ }^{2}$ Departments of Pathology, Chonbuk National University Medical School, Research Institute of Clinical Medicine and Research Institute for Endocrine Sciences, Jeonju, Republic of Korea

Full list of author information is available at the end of the article
}

suppressor [1]. Based on data of cBio Cancer Genomics Portal (http://www.cbioportal.org), the deletion of DBC1 has been reported in $2.5 \%(8 / 316)$ to $7.7 \%(24 / 311)$ of ovarian serous carcinomas [2,3]. Especially, the inhibitory role of DBC1 on SIRT1 supported the possibility that DBC1 could be tumor suppressor because SIRT1 inactivates various tumor suppressors, especially p53 $[4,5]$. However, these findings were followed by conflicting reports, which cast doubt on whether DBC1 is tumor suppressor. In human cancers, the deletion of DBC1 was not a common phenomenon and the balance between SIRT1 and DBC1 was disrupted in human cancers [6]. When the 
interaction between SIRT1 and DBC1 is week, the depletion of DBC1 makes cells susceptible to UV-induced genotoxic stress [7]. Also, DBC1 inhibits senescence of premalignant cells by disrupting the SUV39H1-SIRT1 complex [8]. Moreover, the expression of both DBC1 and SIRT1 were correlated with advanced clinicopathological characteristics and poor prognosis of human malignant tumors [9-16]. In addition, it has been shown that DBC1 has multiple functions involved in the regulation of cell survival, energy metabolism, and intracellular signal transduction [8,12,17-21]. Therefore, DBC1 might have its own role in tumorigenesis in addition to an inhibitory role for SIRT1.

The tumorigenic role of $\mathrm{DBC}$, although controversial, is supported by its role in the inhibition of tumor suppressors $[8,22]$ and activation of nuclear receptors with tumorigenic potential [21]. DBC1 inhibits the tumor suppressor BRCA1 by binding to the BRCT domain in breast cancer [22]. Defect of $B R C A 1 / 2$ is involved in the development of ovarian carcinomas and defects in $B R C A 1 / 2$ are associated with the resistance to platinumbased chemotherapy in ovarian carcinomas [23,24]. Therefore, there is a possibility that $\mathrm{DBC} 1$ may be involved in $B R C A 1 / 2$-related progression of ovarian carcinomas. In addition, inhibition of $\mathrm{DBC} 1$ reduced the proliferation and invasive potential of gastric cancer cells and squamous cell carcinoma cells $[12,20]$. The decrease of tumor invasiveness with inhibition of DBC1 was associated with a decrease in epithelial-mesenchymal transition (EMT) signaling [12].

The role of $\mathrm{DBC} 1$ as a co-activator of hormone receptors raised the possibility that $\mathrm{DBC} 1$ could promote the tumorigenesis of hormone-dependent organs [18,19,21]. In breast carcinoma, $\mathrm{DBC} 1$ is expected to be an indicator of poor prognosis and might be involved in resistance to the estrogen receptor-targeted therapies [14]. However, there has been no study to date on the role of $\mathrm{DBC} 1$ in ovarian tumorigenesis, its relation to BRCA1/2, and the prognostic significance of $\mathrm{DBC} 1$ in ovarian cancers. Therefore, in this study, we evaluated the immunohistochemical expression of $\mathrm{DBC} 1$ and $\mathrm{BRCA} 1$ and their prognostic significance in 104 ovarian carcinomas.

\section{Methods}

\section{Patients and tissue samples}

One hundred and four ovarian carcinomas diagnosed between November 1996 and August 2008, and the original histologic slides, tissue blocks, and clinical information were available were included in the present study. The age of the patients ranged from 21 to 82 years (median; 54 years). All patients received staging operations and 83 patients received platinum- and taxoidbased adjuvant chemotherapy. Among the 83 patients, platinum-resistance was evaluable in 82 patients and 62 patients were sensitive to platinum-based chemotherapy and 20 patients showed platinum-resistance. Platinumresistance was evaluated according to the standard Gynecologic Oncology Group criteria [25]. The patients who experienced recurrence or progression of ovarian cancer within the six months of platinum-based chemotherapy were included in platinum-resistant group. Among the 104 ovarian carcinoma patients, 39 patients experienced relapse and 50 patients died from ovarian carcinoma at the endpoint of follow-up. The five- and ten-year overall survival (OS) rates were $57 \%$ and $50 \%$, respectively. This study was approved by the institutional review board of Chonbuk National University Hospital. Informed consent was provided according to the Declaration of Helsinki.

All histologic slides and clinicopathologic factors were reviewed according to the criteria of the World Health Organization classification of tumors of female reproductive organs [26]. The ovarian carcinomas included in this study, according to the histologic types, were 75 serous carcinomas (12 low-grade serous carcinomas and 63 high-grade serous carcinomas), 20 mucinous carcinomas, 5 endometrioid carcinomas, 3 clear cell carcinomas, and one malignant Brenner tumor. Tumor stage was reviewed according to the guidelines of the tumor, node, and metastasis staging system of the American Joint Committee on Cancer [27]. Thereafter, the ovarian carcinomas were grouped according to their age $(<60$ years versus $\geq$ 60 years), tumor stage (I and II versus III and IV), tumor size $(\leq 10 \mathrm{~cm}$ versus $>10 \mathrm{~cm})$, lymph node metastasis (absence versus presence), presence of ascites (absence versus presence), bilaterality (unilateral versus bilateral), presence of latent distant metastasis during follow-up (absence versus presence), pre-operative serum level of CA19-9 (normal versus elevated, reference value; $0-37 \mathrm{U} / \mathrm{ml}$ ), pre-operative serum level of CA125 (normal versus elevated, reference value; $0-35 \mathrm{U} / \mathrm{ml}$ ), and histologic grade (low; grade 1 versus high; grade 2 and 3). The duration of follow-up ranged from one to 193 months (median; 70 months).

\section{Establishment of tissue microarray and immunohistochemical staining}

Tissue microarray (TMA) established from the most representative solid area with highest histologic grade after review of original H\&E slides. One $5 \mathrm{~mm}$ tissue core per case was used for the construction of a TMA. Immunohistochemical staining for DBC1 (1:100, Bethyl Laboratories, Mongomery, TX) and BRCA1 (1:100, Abcam, Cambridge, MA) was performed on $4 \mu \mathrm{m}$ thick sections on TMA slides. The tissue sections underwent a microwave antigen retrieval procedure in $\mathrm{pH} 6.0$ sodium citrate buffer for 20 minutes. Immunohistochemical analysis was performed by two pathologists (KYJ and KMK) by consensus, without knowledge of the clinicopathological information. For the evaluation of the immunohistochemical staining 
of DBC1 and BRCA1, the Allred nuclear scoring system was used [28]. The staining intensity was scored as 0 (no staining), 1 (weak staining), 2 (intermediate staining), or 3 (strong staining). The area of staining was scored as 0 (no staining), 1 ( $1 \%$ of the cells stained positive), 2 (2-10\% of the cells stained positive), 3 (11-33\% of cells stained positive), 4 (34-66\% of cells stained positive), or 5 (67-100\% of cells stained positive). Thereafter, the sum score was obtained by adding the intensity score and staining area score $[13,29]$, to give maximum sum score of eight and a minimum sum score of zero.

\section{Statistical analysis}

The cut-off point of the immunohistochemical staining score for DBC1 and BRCA1 expression was determined by receiver operating characteristic curve analysis at the highest positive likelihood ratio point for the event of OS. The cut-off points for the sum score for DBC1 and BRCA1 were seven and six, respectively. The immunostaining for $\mathrm{DBC} 1$ was considered positive when the sum score was greater than or equal to seven and BRCA1 expression was considered positive when the sum score was greater than or equal to six. The endpoint of interest was OS and relapse-free survival (RFS). The endpoint of follow-up was the date of last contact or date of death of patients through August 2013. OS was calculated as the time from the date of diagnosis to the date of last contact or death from ovarian carcinomas. The patients who were alive at last contact were treated as censored for OS analysis. RFS calculated from the date of diagnosis to the date of last contact, local relapse, latent metastasis, or death from ovarian carcinomas. The patients who were alive at last contact without experience of local relapse or latent metastasis were treated as censored for RFS analysis. Survival analyses were performed according to the Kaplan-Meier method, and univariate and multivariate
Cox proportional hazard regression analysis. The association between immunohistochemical positivity of $\mathrm{DBC} 1$ expression and other clinicopathological factors potentially predictive of prognosis were analyzed using Pearson's chi-square test. SPSS software (version 20.0) was used throughout and $P$-values less than 0.05 were considered statistically significant.

\section{Results \\ Association of DBC1 and BRCA1 expression with clinicopathologic characteristics of ovarian carcinoma patients}

As shown in Figure 1, DBC1 was expressed exclusively in the nuclei of tumor cells and nuclear expression was evaluated for the evaluation of DBC1. BRCA1 was expressed in both the cytoplasm and nuclei; however, we have evaluated only for the nuclear expression [30,31]. The associations between the expression of DBC1, BRCA1, and variable clinicopathologic features are summarized in Table 1. Positive expression of DBC1 and BRCA1 were seen in 63\% (66/104) and 44\% (46/104) of overall ovarian carcinomas, respectively. Expression of DBC1 was significantly associated with higher tumor stage $(P=0.015)$, bilateral tumors $(P=0.008)$, latent distant metastasis $(P=$ 0.016), platinum-resistance $(P=0.016)$, higher pre-operative serum level of CA125 $(P=0.004)$, higher histologic grade $(P<0.001)$, histologic type of ovarian carcinoma $(P<$ $0.001)$, and BRCA1 expression $(P<0.001)$. DBC1 was expressed in $72 \%(54 / 75)$ of serous carcinoma and $100 \%$ of endometrioid carcinoma (5/5), clear cell carcinoma $(3 / 3)$, and the malignant Brenner tumor (1/1). However, only $15 \%(3 / 20)$ of mucinous carcinoma were positive for DBC1. BRCA1 expression was significantly associated with latent distant metastasis $(P=0.012)$, platinum-resistance $(P=0.014)$, and higher histologic grade $(P=0.007)$.

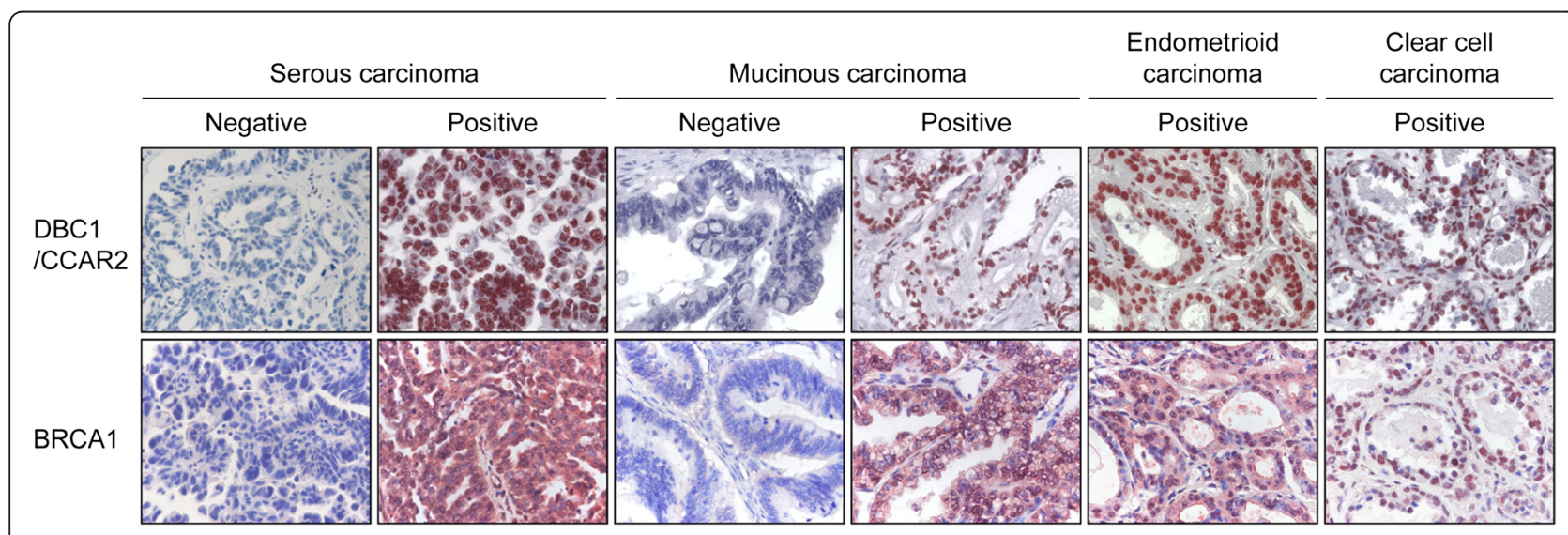

Figure 1 Immunohistochemical expression of DBC1/CCAR2 and BRCA1 in ovarian carcinomas. DBC1/CCAR2 is primarily expressed in the nuclei of the tumor cells. BRCA1 is expressed both in the cytoplasm and nuclei of the tumor cells. Original magnification; $\times 400$. 
Table 1 Clinicopathological variables and the expression of DBC1 and BRCA1 in ovarian carcinomas

\begin{tabular}{|c|c|c|c|c|c|c|c|c|c|c|c|}
\hline \multicolumn{2}{|l|}{ Characteristics } & No. & \multicolumn{2}{|c|}{ Overall ovarian car } & \multicolumn{2}{|l|}{ cinomas } & No. & \multicolumn{2}{|l|}{ Serous C } & \multicolumn{2}{|c|}{ Arcinomas } \\
\hline \multirow[t]{2}{*}{ Age, y } & $<60$ & 71 & $41(58 \%)$ & 0.076 & $28(39 \%)$ & 0.149 & 45 & $29(64 \%)$ & 0.074 & $20(44 \%)$ & 0.450 \\
\hline & $\geq 60$ & 33 & $25(76 \%)$ & & $18(55 \%)$ & & 30 & 25 (83\%) & & $16(53 \%)$ & \\
\hline \multirow[t]{2}{*}{ Stage } & $|\&| \mid$ & 52 & 27 (52\%) & 0.015 & $21(40 \%)$ & 0.430 & 29 & 18 (62\%) & 0.128 & $13(45 \%)$ & 0.662 \\
\hline & III \& IV & 52 & $39(75 \%)$ & & $25(48 \%)$ & & 46 & $36(78 \%)$ & & $23(50 \%)$ & \\
\hline \multirow[t]{2}{*}{ Tumor size, cm } & $\leq 10$ & 68 & 48 (71\%) & 0.038 & $31(46 \%)$ & 0.702 & 56 & 40 (71\%) & 0.850 & $26(46 \%)$ & 0.64 \\
\hline & $>10$ & 36 & 18 (50\%) & & $15(42 \%)$ & & 19 & $14(74 \%)$ & & $10(53 \%)$ & \\
\hline \multirow[t]{2}{*}{ LN metastasis } & Absence & 84 & $50(60 \%)$ & 0.087 & $37(44 \%)$ & 0.939 & 56 & 39 (70\%) & 0.435 & $28(50 \%)$ & 0.552 \\
\hline & Presence & 20 & 16 (80\%) & & $9(45 \%)$ & & 19 & 15 (79\%) & & $8(42 \%)$ & \\
\hline \multirow[t]{2}{*}{ Ascites } & Absence & 71 & $41(58 \%)$ & 0.076 & $28(39 \%)$ & 0.149 & 45 & 31 (69\%) & 0.462 & $18(40 \%)$ & 0.089 \\
\hline & Presence & 33 & $25(76 \%)$ & & $18(55 \%)$ & & 30 & $23(77 \%)$ & & $18(60 \%)$ & \\
\hline \multirow[t]{2}{*}{ Bilaterality } & Unilateral & 59 & 31 (53\%) & 0.008 & $24(41 \%)$ & 0.404 & 34 & $21(62 \%)$ & 0.072 & $16(47 \%)$ & 0.882 \\
\hline & Bilateral & 45 & 35 (78\%) & & $22(49 \%)$ & & 41 & $33(80 \%)$ & & $20(49 \%)$ & \\
\hline \multirow[t]{2}{*}{ LD Meta } & Absence & 76 & $43(57 \%)$ & 0.016 & $28(37 \%)$ & 0.012 & 52 & $34(65 \%)$ & 0.055 & $21(40 \%)$ & 0.047 \\
\hline & Presence & 28 & $23(82 \%)$ & & $18(64 \%)$ & & 23 & 20 (87\%) & & $15(65 \%)$ & \\
\hline \multirow[t]{2}{*}{ Platinum-resistance } & Absence & 62 & $38(61 \%)$ & 0.016 & $21(34 \%)$ & 0.014 & 48 & $32(67 \%)$ & 0.008 & $19(40 \%)$ & 0.043 \\
\hline & Presence & 20 & $18(90 \%)$ & & $13(65 \%)$ & & 16 & $16(100 \%)$ & & 11 (69\%) & \\
\hline \multirow[t]{2}{*}{ CA19-9 } & Normal & 66 & 47 (71\%) & 0.060 & $32(48 \%)$ & 0.158 & 50 & 39 (78\%) & 0.341 & $26(52 \%)$ & 0.156 \\
\hline & Elevated & 17 & $8(47 \%)$ & & $5(29 \%)$ & & 8 & $5(63 \%)$ & & $2925 \%)$ & \\
\hline \multirow[t]{2}{*}{ CA125 } & Normal & 18 & 7 (39\%) & 0.004 & $5(28 \%)$ & 0.121 & 7 & $4(57 \%)$ & 0.249 & $4(57 \%)$ & 0.630 \\
\hline & Elevated & 75 & $56(75 \%)$ & & $36(48 \%)$ & & 61 & 47 (77\%) & & $29(48 \%)$ & \\
\hline \multirow[t]{2}{*}{ Histologic grade } & Low (1) & 27 & $5(19 \%)$ & $<0.001$ & $6(22 \%)$ & 0.007 & 12 & $4(33 \%)$ & 0.001 & $4(33 \%)$ & 0.267 \\
\hline & High (2 \& 3) & 77 & $61(79 \%)$ & & $40(52 \%)$ & & 63 & $50(79 \%)$ & & $32(51 \%)$ & \\
\hline \multirow[t]{5}{*}{ Histologic type } & Serous & 75 & $54(72 \%)$ & $<0.001$ & $36(48 \%)$ & 0.254 & & & & & \\
\hline & Mucinous & 20 & $3(15 \%)$ & & $5(25 \%)$ & & & & & & \\
\hline & Endometrioid & 5 & $5(100 \%)$ & & $3(60 \%)$ & & & & & & \\
\hline & Clear cell & 3 & $3(100 \%)$ & & $1(33 \%)$ & & & & & & \\
\hline & Malignant Brenner & 1 & $1(100 \%)$ & & $1(100 \%)$ & & & & & & \\
\hline \multirow[t]{2}{*}{ BRCA1 } & Negative & 58 & $28(48 \%)$ & $<0.001$ & & & 39 & $22(56 \%)$ & 0.002 & & \\
\hline & Positive & 46 & 38 (83\%) & & & & 36 & $32(89 \%)$ & & & \\
\hline
\end{tabular}

LN; lymph node, LD Meta; latent distant metastasis.

The cases included in this study are heterogeneous. Various histologic types of ovarian carcinomas with different biologic and clinical background were included in this study. Thus, we did additional analysis according to the histologic types, especially for the serous carcinoma. Among the 75 serous carcinomas, DBC1 expression was significantly associated with higher histologic grade $(P=0.001)$ and platinum-resistance $(P=0.008)$ and showed a slight association of borderline significance with bilateral tumor $(P=0.072)$ and presence of latent distant metastasis $(P=0.055)$. BRCA1 expression was significantly associated with latent distant metastasis $(P=0.047)$ and platinum-resistance $(P=0.008)$ (Table 1).
Expression of DBC1 and BRCA1 correlate with reduced overall survival and relapse-free survival in ovarian carcinomas by univariate analysis

Univariate analysis for the OS and RFS of the variable clinicopathological factors and DBC 1 and BRCA1 expression in ovarian carcinomas are shown in Table 2. Among the 104 general cases of ovarian carcinoma, older age of the patients (OS; $P<0.001$, RFS; $P=0.003$ ), higher tumor stage (OS; $P<0.001$, RFS; $P<0.001$ ), presence of ascites (OS; $P=0.006$, RFS; $P=0.017$ ), increased pre-operative serum level of CA125 (OS; $P=0.013$, RFS; $P=0.004$ ), histologic grade (OS; $P=0.005$, RFS; $P<0.001$ ), DBC1 expression (Log-rank, OS; $P<0.001$, RFS; $P<0.001$ ), and BRCA1 expression (Log-rank, OS; $P=0.003$, RFS; $P=$ 
Table 2 Univariate Cox regression analyses for overall survival and relapse-free survival in ovarian carcinomas

\begin{tabular}{|c|c|c|c|c|c|}
\hline \multirow[t]{2}{*}{ Characteristics } & \multirow[t]{2}{*}{ No. } & \multicolumn{2}{|l|}{ OS } & \multicolumn{2}{|l|}{ RFS } \\
\hline & & HR (95\% Cl) & $P$ & HR $(95 \% \mathrm{Cl})$ & $P$ \\
\hline \multicolumn{6}{|l|}{ Overall ovarian carcinomas $(n=104)$} \\
\hline Age, $y, \geq 60(v s<60)$ & $33 / 104$ & $2.686(1.535-4.698)$ & $<0.001$ & $2.157(1.299-3.582)$ & 0.003 \\
\hline Stage, III \& IV (vs I \& II) & $52 / 104$ & $3.579(1.917-6.681)$ & $<0.001$ & $3.930(2.245-6.878)$ & $<0.001$ \\
\hline Tumor size, $\mathrm{cm},>10$ (vs $\leq 10)$ & $36 / 104$ & $0.524(0.274-1.004)$ & 0.051 & $0.649(0.375-1.125)$ & 0.123 \\
\hline LN metastasis, presence (vs absence) & $20 / 104$ & $1.568(0.831-2.959)$ & 0.165 & $1.889(1.076-3.314)$ & 0.027 \\
\hline Ascites, presence (vs absence) & $33 / 104$ & $1.997(1.140-3.498)$ & 0.016 & $1.854(1.116-3.077)$ & 0.017 \\
\hline Bilaterality, bilateral (vs unilateral) & $45 / 104$ & $1.647(0.943-2.876)$ & 0.080 & $1.995(1.204-3.303)$ & 0.007 \\
\hline CA19-9, elevated (vs normal) & $17 / 83$ & $0.753(0.314-1.806)$ & 0.525 & $0.811(0.379-1.733)$ & 0.588 \\
\hline CA125, elevated (vs normal) & 75/93 & $4.451(1.376-14.393)$ & 0.013 & $4.458(1.609-12.351)$ & 0.004 \\
\hline Histologic grade, high (vs low) & 77/104 & $3.762(1.491-9.496)$ & 0.005 & $3.794(1.719-8.374)$ & $<0.001$ \\
\hline DBC1, positive (vs negative) & $66 / 104$ & $3.474(1.684-7.166)$ & $<0.001$ & $3.007(1.624-5.567)$ & $<0.001$ \\
\hline BRCA1, positive (vs negative) & $46 / 104$ & $2.263(1.287-3.979)$ & 0.005 & $2.254(1.359-3.739)$ & 0.002 \\
\hline \multicolumn{6}{|l|}{ Serous carcinomas $(n=75)$} \\
\hline Age, $y, \geq 60(v s<60)$ & $30 / 75$ & $2.601(1.411-4.796)$ & 0.002 & $1.926(1.116-3.323)$ & 0.019 \\
\hline Stage, III \& IV (vs I \& II) & $46 / 75$ & $2.263(1.131-4.528)$ & 0.021 & $2.809(1.485-5.312)$ & 0.001 \\
\hline Tumor size, $\mathrm{cm},>10$ (vs $\leq 10)$ & $19 / 75$ & $0.625(0.289-1.352)$ & 0.233 & $0.817(0.436-1.532)$ & 0.529 \\
\hline LN metastasis, presence (vs absence) & $19 / 75$ & $1.200(0.613-2.349)$ & 0.595 & $1.502(0.831-2.714)$ & 0.178 \\
\hline Ascites, presence (vs absence) & $30 / 75$ & $1.793(0.977-3.291)$ & 0.060 & $1.645(0.953-2.839)$ & 0.074 \\
\hline Bilaterality, bilateral (vs unilateral) & $41 / 75$ & $1.200(0.647-2.224)$ & 0.563 & $1.423(0.813-2.492)$ & 0.217 \\
\hline CA19-9, elevated (vs normal) & $8 / 58$ & $0.679(0.206-2.236)$ & 0.524 & $1.086(0.425-2.773)$ & 0.863 \\
\hline CA125, elevated (vs normal) & $61 / 68$ & $2.898(0.697-12.056)$ & 0.144 & $2.152(0.668-6.931)$ & 0.199 \\
\hline Histologic grade, high (vs low) & $63 / 75$ & $4.401(1.061-18.260)$ & 0.041 & $4.341(1.347-13.990)$ & 0.014 \\
\hline DBC1, positive (vs negative) & $54 / 75$ & $4.277(1.674-10.926)$ & 0.002 & $2.811(1.363-5.794)$ & 0.005 \\
\hline BRCA1, positive (vs negative) & $36 / 75$ & $1.861(1.007-3.438)$ & 0.047 & $1.792(1.032-3.110)$ & 0.038 \\
\hline \multicolumn{6}{|l|}{ High-grade serous carcinomas $(n=63)$} \\
\hline Age, $y, \geq 60(v s<60)$ & $27 / 63$ & $2.090(1.119-3.900)$ & 0.021 & $1.591(0.907-2.791)$ & 0.105 \\
\hline Stage, III \& IV (vs I \& II) & $38 / 63$ & $2.188(1.080-4.434)$ & 0.030 & $2.811(1.464-5.399)$ & 0.002 \\
\hline Tumor size, $\mathrm{cm},>10(v s \leq 10)$ & $15 / 63$ & $0.736(0.339-1.599)$ & 0.439 & $0.860(0.448-1.651)$ & 0.650 \\
\hline LN metastasis, presence (vs absence) & $15 / 63$ & $1.520(0.766-3.017)$ & 0.232 & $1.841(0.992-3.417)$ & 0.053 \\
\hline Ascites, presence (vs absence) & $26 / 63$ & $1.395(0.749-2.598)$ & 0.294 & $1.340(0.764-2.351)$ & 0.308 \\
\hline Bilaterality, bilateral (vs unilateral) & $36 / 63$ & $1.300(0.683-2.475)$ & 0.424 & $1.452(0.809-2.603)$ & 0.211 \\
\hline CA19-9, elevated (vs normal) & $6 / 49$ & $0.726(0.220-2.403)$ & 0.600 & $1.344(0.521-3.467)$ & 0.541 \\
\hline CA125, elevated (vs normal) & $53 / 58$ & $2.220(0.531-9.276)$ & 0.275 & $1.443(0.446-4.672)$ & 0.540 \\
\hline DBC1, positive (vs negative) & $50 / 63$ & $4.031(1.427-11.382)$ & 0.008 & $2.540(1.135-5.684)$ & 0.023 \\
\hline BRCA1, positive (vs negative) & $32 / 63$ & $2.010(1.063-3.803)$ & 0.032 & $1.719(0.973-3.038)$ & 0.062 \\
\hline
\end{tabular}

0.003) were associated with shorter OS and RFS (Table 2) (Figure 2). The patients with tumors expressing DBC1 had a 3.474-fold $(P<0.001,95 \%$ confidence interval [95\% $\mathrm{CI}]$; 1.684-7.166) greater risk of death and a 3.007 -fold $(P<0.001$, 95\% CI; 1.624-5.567) greater risk of relapse or death. The five- and ten-year OS rates of the DBC1negative group were $78 \%$ and $73 \%$, respectively, and were only $42 \%$ and $36 \%$ in DBC1-positive group, respectively. In addition, among the 83 patients who received adjuvant chemotherapy, DBC1 expression was significantly associated with shorter OS $(P=0.004$, hazard ration [HR]; 3.625, 95\% CI; $1.518-8.656)$ and RFS $(P=0.004, \mathrm{HR} ; 2.738,95 \%$ CI; 1.368-5.477). BRCA1 expression predicted shorter OS $(P=0.005, \mathrm{HR} ; 2.263,95 \% \mathrm{CI} ; 1.287-3.979)$ and RFS $(P=0.002$, HR; 2.254, 95\% CI; 1.359-3.739) (Table 2).

When we did additional analysis in the subpopulation of serous carcinomas, the factors significantly associated with both OS and RFS by univariate analysis were the 

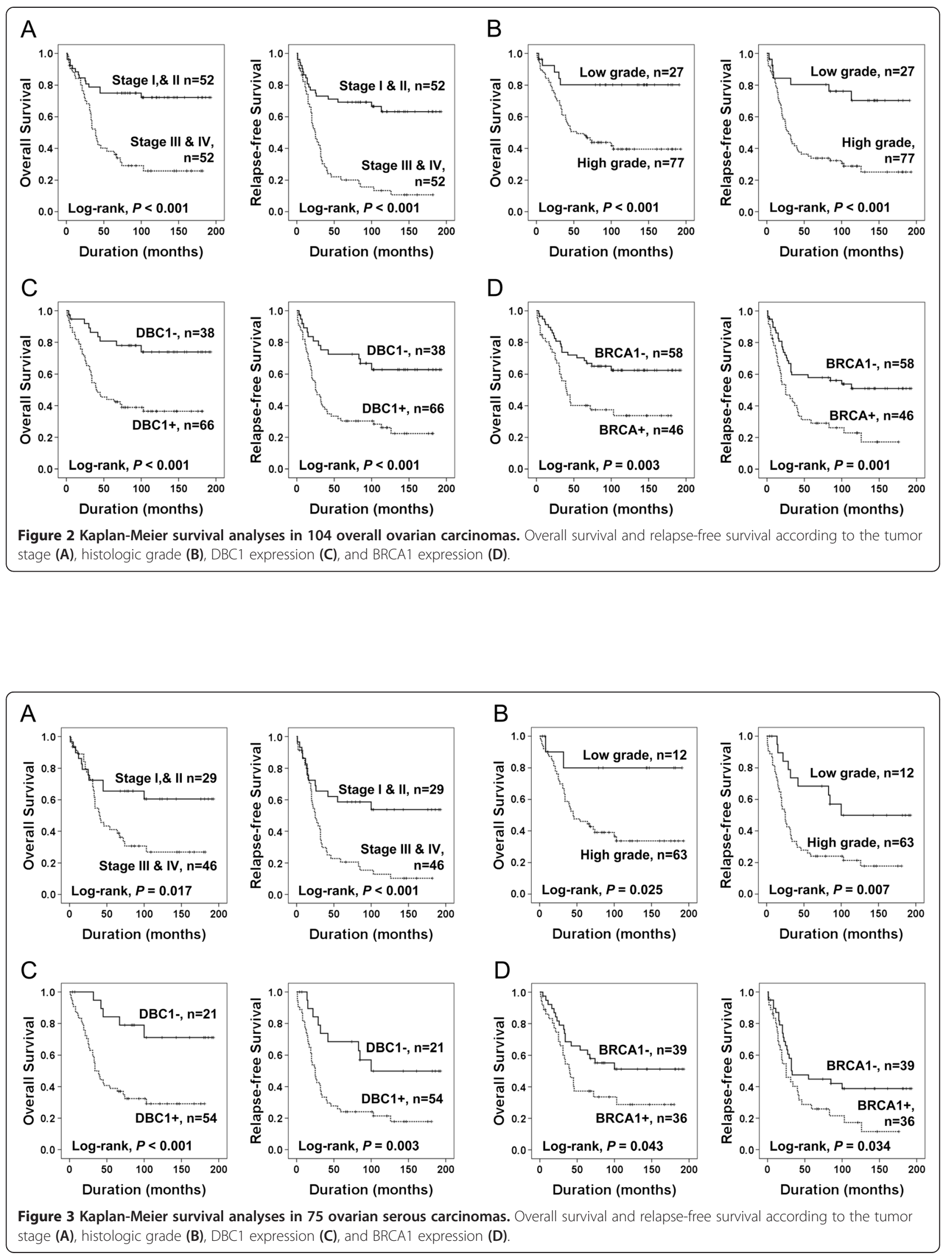
age of patients (OS; $P=0.002$, RFS; $P=0.019$ ), tumor stage (OS; $P=0.021$, RFS; $P=0.001$ ), histologic grade (OS; $P=0.041$, RFS; $P=0.014$ ), and DBC1 expression (Log-rank, OS; $P<0.001$, RFS; $P=0.003$ ), and BRCA1 expression (Log-rank, OS; $P=0.043$, RFS; $P=0.034$ ) (Table 2) (Figure 3). The patients who had DBC1-expressing serous carcinoma had a 4.277 -fold $(P=0.002$, 95\% CI; 1.674 10.926) greater risk of death and its expression was significantly associated with shorter RFS ( $P=0.005$, HR; 2.811, 95\% CI; 1.363-5.794) (Figure 3C).

Among the 63 high-grade serous carcinomas, tumor stage and the expression of $\mathrm{DBC} 1$ were significantly associated with both OS and RFS, and the age of patients and BRCA1 expression were significantly associated with OS (Table 2) (Figure 4). The expression of DBC1 predicted a 4.031-fold $(P=0.008,95 \%$ CI; $1.427-11.382)$ greater risk of death and a 2.540 -fold $(P=0.005,95 \% \mathrm{CI}$; 1.135-5.684) greater risk of relapse or death of highgrade serous carcinoma patients (Table 2). The OS rates at five- and ten-years of DBC1-negative high-grade serous carcinomas were $85 \%$ and $64 \%$, respectively, and were $36 \%$ and $25 \%$ in DBC1-positive high-grade serous carcinomas.

In the 20 cases of mucinous carcinomas, tumor stage was significantly associated with shorter OS (Log-rank, $P<$ 0.001 ) and RFS (Log-rank, $P<0.001$ ). DBC1 expression
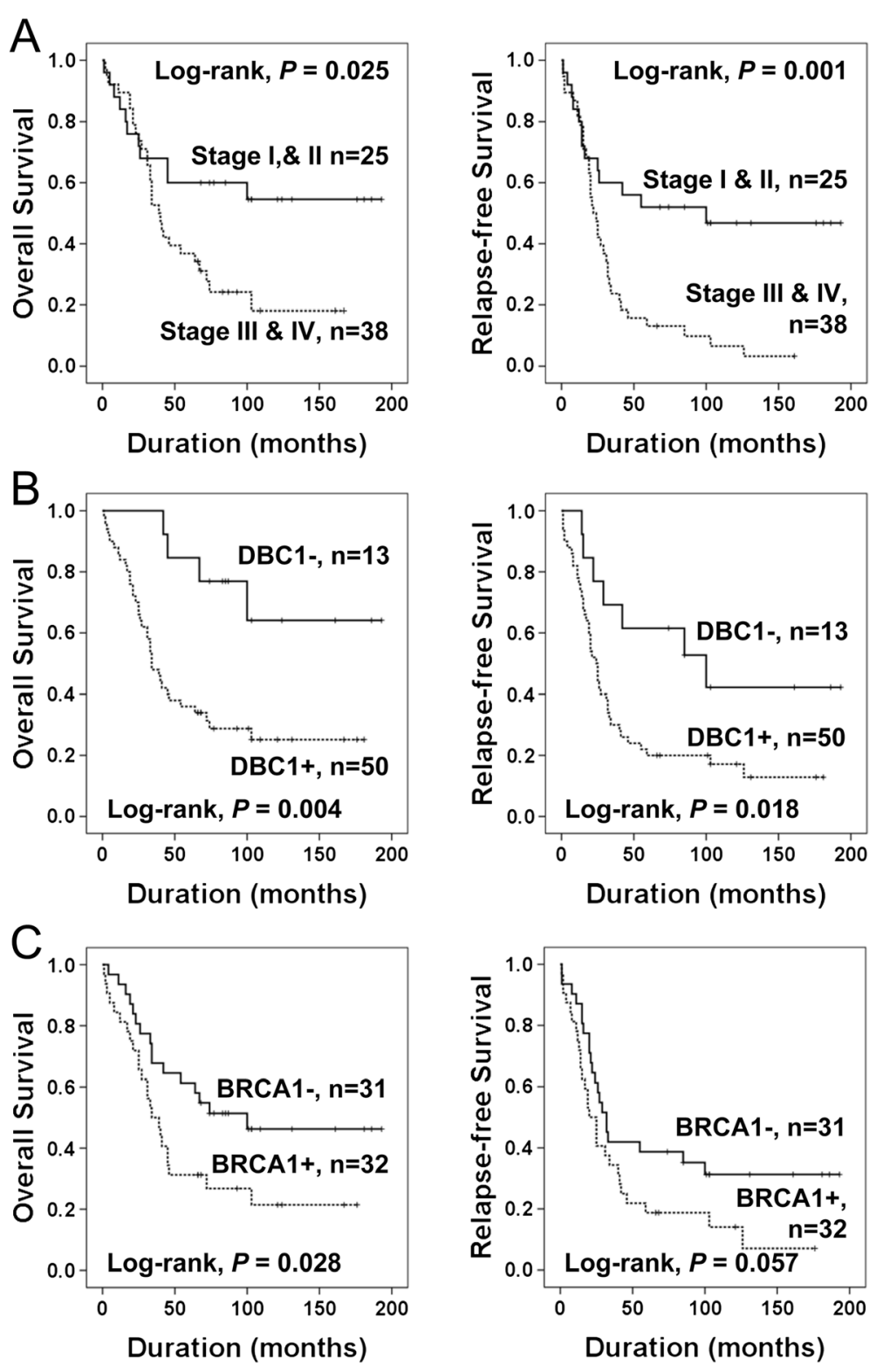

Figure 4 Kaplan-Meier survival analyses in 63 high-grade serous carcinomas. Overall survival and relapse-free survival according to the tumor stage (A), DBC1 expression (B), and BRCA1 expression (C). 
showed borderline significance for the prediction of OS of mucinous carcinoma patients (Log-rank, $P=0.060$ ).

\section{Expression of DBC1 is an independent prognostic indicator of worse survival outcome in ovarian carcinomas by multivariate analysis}

The factors significantly associated with OS or RFS by univariate analysis were included in the multivariate analysis. Because of data for CA125 was missing in 11 patients, the CA125 level was not included in the multivariate analysis. The factors included in the multivariate analysis of OS and RFS were age, tumor stage, lymph node metastasis, presence of ascites, bilaterality of the tumor, histologic grade, BRCA1 expression, and DBC1 expression. In 104 ovarian carcinomas, the factors significantly associated with $\mathrm{OS}$ by multivariate analysis were age $(P=0.010)$, tumor stage $(P=0.006)$, and DBC1 expression $(P=0.021)$. The patients with tumors expressing DBC1 had a 2.423 fold (95\% CI, 1.144-5.132) greater risk of death (Table 3). Tumor stage $(P<0.001)$ and histologic grade $(P=0.007)$ were the independent predictor of RFS. In addition, DBC1 expression was an independent predictor of OS $(P=0.026$, HR; 2.735, 95\% CI; 1.128-6.634) among the 83 patients who received adjuvant chemotherapy, as indicated by multivariate analysis.

In the subpopulation of 75 serous carcinomas, the age of the patients $(P=0.010)$ and $\mathrm{DBC} 1$ expression $(P=0.006)$ were the independent predictors of OS. The expression of DBC1 predicted a 3.757-fold (95\% CI, 1.462-9.653) greater risk of death. The factors significantly associated with RFS in serous carcinoma were tumor stage $(P<0.001)$ and histologic grade $(P=0.009)$ (Table 3$)$. In addition, the age of the patients $(P=0.035)$ and the expression of DBC1 $(P=0.011)$ were the independent predictor of $\mathrm{OS}$ in high-grade serous carcinomas. The expression of DBC1 predicted a 3.828 -fold (95\% CI, 1.353-10.827) greater risk of death of high-grade serous carcinoma patients (Table 3).

\section{Discussion}

This study has shown that immunohistochemical expression of DBC1 was significantly associated with advanced clinicopathological factors of ovarian carcinoma such as higher tumor stage, latent distant metastasis, platinum-resistance, elevated serum level of CA125, and higher histologic grade. Moreover, DBC1 expression was significantly associated with shorter survival of ovarian carcinomas, especially in high-grade serous carcinomas. In agreement with our results, increased expression of DBC1 has been reported as an indicator of poor prognosis of gastric carcinoma [15], breast carcinoma [14], colorectal carcinoma [9], esophageal carcinoma [20], clear cell renal cell carcinoma [12], diffuse large B cell lymphoma [13], and soft tissue sarcomas [13]. Nevertheless, there has been no report investigating $\mathrm{DBC} 1$ expression in ovarian carcinomas. Therefore, this is the first report that examined DBC1 expression in human ovarian tumors and suggests that $\mathrm{DBC} 1$ expression might be usable as a prognostic indicator for ovarian carcinoma patients.

DBC1 is interesting because of its putative role for the inhibition of SIRT1 and has been suggested as tumor suppressor $[4,16,32]$. However, there have been conflicting reports regarding the role of DBC1 and SIRT1 in human

Table 3 Multivariate Cox regression analyses for overall survival and relapse-free survival in ovarian carcinomas

\begin{tabular}{|c|c|c|c|c|}
\hline \multirow[t]{2}{*}{ Characteristics } & \multicolumn{2}{|l|}{ OS } & \multicolumn{2}{|l|}{ RFS } \\
\hline & HR $(95 \% \mathrm{Cl})$ & $P$ & HR $(95 \% \mathrm{Cl})$ & $P$ \\
\hline \multicolumn{5}{|l|}{ Overall ovarian carcinomas ${ }^{a}$} \\
\hline Age, $y, \geq 60(v s<60)$ & $2.100(1.191-3.702)$ & 0.010 & & \\
\hline Stage, III \& IV (vs I \& II) & $2.488(1.295-4.780)$ & 0.006 & $3.426(1.944-6.036)$ & $<0.001$ \\
\hline Histologic grade, high (vs low) & & & $3.023(1.355-6.742)$ & 0.007 \\
\hline DBC1, positive (vs negative) & $2.423(1.144-5.132)$ & 0.021 & & \\
\hline \multicolumn{5}{|l|}{ Serous carcinomas ${ }^{b}$} \\
\hline Age, $y, \geq 60$ (vs < 60) & $2.241(1.212-4.144)$ & 0.010 & & \\
\hline Stage, III \& IV (vs I \& II) & & & $3.023(1.588-5.755)$ & $<0.001$ \\
\hline Histologic grade, high (vs low) & & & $4.818(1.487-15.614)$ & 0.009 \\
\hline DBC1, positive (vs negative) & $3.757(1.462-9.653)$ & 0.006 & & \\
\hline \multicolumn{5}{|l|}{ High-grade serous carcinomas ${ }^{c}$} \\
\hline Age, $y, \geq 60$ (vs < 60) & $1.961(1.050-3.664)$ & 0.035 & & \\
\hline Stage, III \& IV (vs I \& II) & & & $2.488(1.280-4.836)$ & 0.007 \\
\hline DBC1, positive (vs negative) & $3.828(1.353-10.827)$ & 0.011 & $2.059(0.907-4.677)$ & 0.084 \\
\hline
\end{tabular}

${ }^{a}$ The variables included in the multivariate analysis were age, tumor stage, lymph node metastasis, presence of ascites, bilaterality of the tumor, histologic grade, BRCA1 expression, and DBC1 expression. ${ }^{\mathrm{b}}$ The variables included in the multivariate analysis were age, tumor stage, histologic grade, BRCA1 expression, and DBC1 expression. 'The variables included in the multivariate analysis were age, tumor stage, BRCA1 expression, and DBC1 expression. 
cancers. SIRT1-induced expression of various oncogenes and formed a positive-feedback loop with the c-Myc oncogene to stimulate tumorigenesis [33,34]. However, SIRT1 formed a negative-feedback loop with the c-Myc oncogene in another report [35]. Moreover, the effects of the expression of DBC1 and SIRT1 in human malignant tumors varied according to cell type $[6,9,34,36,37]$. The expression of both DBC1 and SIRT1 predicted shorter survival of gastric carcinoma [15], breast carcinoma [14], clear cell renal cell carcinoma [12], soft-tissue sarcoma [13], and diffuse large B cell lymphoma $[13,16]$. In colon cancer, DBC1 was overexpressed in colorectal cancer and predicted shorter survival of patients [9]. In contrast, another study reported that SIRT1 expression is associated with poor prognosis but $\mathrm{DBC} 1$ expression is associated with favorable prognosis of gastric cancer patients [36]. Although the expression of SIRT1 was higher in ovarian carcinomas compared with benign and borderline ovarian tumors, SIRT1 expression was associated favorable prognosis of ovarian carcinoma patients [38]. Therefore, poor prognosis of DBC1-expressing ovarian carcinoma might be related to its inhibitory role for SIRT1. However, the relationship between DBC1 and SIRT1 was been frequently dissociated as shown in breast cancer [6]. Moreover, the knock-down of DBC1 inhibited proliferation of liver cancer cells [37] and suppressed invasiveness of gastric cancer cells [12]. Therefore, it may be likely that $\mathrm{DBC} 1$ has its own role in tumorigenesis. $\mathrm{DBC} 1$ regulates BRCA1-mediated function by binding to the BRCT domain in addition to the suppression of SIRT1 expression [22]. In addition, $\mathrm{DBC1}$ inhibits senescence of premalignant cells by disrupting the SUV39H1-SIRT1 complex. However, DBC1 showed a co-inhibitory effect for SUV39H1 and SIRT1 [8]. Therefore, there is a possibility that DBC1 may have both tumorigenic and anti-tumorigenic roles $[8,22]$. Thus, further study is needed to explore the exact role of $\mathrm{DBC} 1$ in tumorigenesis.

Because the expression of DBC1 was positively correlated with higher tumor stage, higher tumor grade, and latent metastasis of ovarian carcinoma, there is a possibility that DBC1 might be involved in the acquisition of invasive and metastatic potential. Recently, it has been shown that $\mathrm{DBC} 1$ is associated with the invasive potential of esophageal squamous cell carcinoma [20] and is important in the EMT of gastric carcinoma cells [12]. Especially, the oncogenic role of $\mathrm{DBC} 1$ was regulated by the kinase effect of $\mathrm{CK} 2 \alpha$. CK2 $\alpha$ phosphorylates DBC1 and that is important for the induction of EMT. Knockdown of DBC1 inhibited invasiveness of gastric cancer cells and a point mutation at the phosphorylation site of $\mathrm{DBC} 1 \mathrm{de}-$ creased the expression of MMP2, MMP9, snail, smad3, and $\mathrm{N}$-cadherin [12]. In addition, $\mathrm{DBC} 1$ induced anoikis resistance that is important in tumor metastasis by activating the NFkB signaling pathway in breast cancer [17].
The higher rate of distant metastatic relapse in DBC1expressing ovarian carcinomas raises the possibility that DBC1 might be involved in the acquisition of resistance for the postoperative chemotherapies. Among the 83 ovarian carcinoma patients who received adjuvant chemotherapy, the expression of DBC1 predicted shorter OS and RFS. In addition, among the 55 high-grade serous carcinoma patients who received adjuvant chemotherapy, DBC1 expression was significantly associated with poor OS $(P=$ 014, HR; 4.484, 95\% CI; $1.362-14.758)$ and RFS $(P=0.042$, HR; 2.471, 95\% CI; 1.035-5.899). Moreover, DBC1 expression correlated with platinum-resistance. All serous carcinoma patients having tumors with DBC1 expression $(100 \%, 16 / 16)$ showed platinum-resistance. In contrast, $62 \%(32 / 48)$ of DBC1-negative serous carcinoma patients showed platinum-resistance. Similarly, DBC1 expression was associated with frequent relapse and shorter survival of breast carcinoma patients who received adjuvant chemotherapy [14]. Although it did not reach statistical significance, lower expression of DBC1 would indicate a favorable pathological response to chemotherapy [39].

Because $\mathrm{DBC1}$ is involved in the inhibition of BRCA1 [22] and BRCA1/2 status is important in the development and progression of ovarian carcinomas, we investigated the immunohistochemical expression of BRCA1 in ovarian carcinomas. Although we could evaluate the immunohistochemical expression of BRCA1, a recent report has shown that there is a strong correlation between the immunohistochemical expression and molecular events in BRCA1 [30]. In this study, BRCA1 expression was significantly associated with latent distant metastasis, platinumresistance, and higher histologic grade. In addition, in agreement with previous reports $[24,40,41]$, we have demonstrated that low-expression of BRCA1 is associated with poor survival of ovarian carcinomas. The reason why the patients with defective BRCA1/2 have a longer survival times compared with $B R C A 1 / 2$ wild-type carcinomas is related with $B R C A 1 / 2$-defects in cells making them more sensitive to conventional chemotherapy, especially to the platinum-based chemotherapy $[23,24,42]$. Our results have also shown that BRCA1-positivity is significantly associated with increased platinum-resistance (Table 1). Thereby, several therapeutic applications according to the $B R C A 1 / 2$ status are under evaluation. Recently, PARP inhibitors have been reported as being specifically applicable to the treatment of $B R C A 1 / 2$-defective cancers $[43,44]$. In our study, the expression of DBC1 and BRCA1 showed positive correlation and the expressions of both molecules was related with platinum-resistance and shorter survival of ovarian carcinoma patients. Based on the inhibitory role of DBC1 for the BRCA1 [22], the co-expressing pattern of these two molecules in the poor prognostic group of ovarian carcinomas is questionable and paradoxical as $B R C A 1 / 2$ defective cancers are more susceptible to therapy 
$[23,24,42]$. This phenomenon might be related with the diverse roles of DBC1 in tumorigenesis and further study is needed.

Another possible oncogenic role of $\mathrm{DBC} 1$ might be related with its role in the co-activation of nuclear receptors $[18,19,21]$. DBC1 co-activates estrogen receptor and androgen receptor (AR), which can be ligand-dependent or ligand-independent $[18,19,21]$. Although there was no significant correlation between the expression of $\mathrm{DBC} 1$ and estrogen receptor in breast carcinoma [14], significant positive correlations between the expression of $\mathrm{DBC} 1$ and $\mathrm{AR}$ have been reported in clear cell renal cell carcinoma [12] and diffuse large B cell lymphoma [11]. Especially, the expression of both DBC1 and AR predicted shorter survival of cancer patients [11,12,14-16]. Additionally, ovarian epithelium expressing AR and androgen induced proliferation of ovarian epithelial cells and inhibited cell death [45]. In ovarian high-grade serous carcinomas, immunohistochemical expression of AR correlated with the S-phase fraction and AR expression decreased with platinum-based chemotherapy [46]. These reports suggest that AR is involved in the ovarian carcinogenesis. Therefore, there is a possibility that DBC1 is involved in ovarian tumorigenesis with the interaction with AR and further study is needed.

Ovarian carcinomas are a heterogeneous group of cancers that have origins and pathogenic profiles that differ according to histologic types. Therefore, when we consider the prognostic impact of DBC1 expression according to histologic types of ovarian carcinoma, $\mathrm{DBC} 1$ predicted shorter survival in serous carcinomas, especially in highgrade serous carcinomas. The 10 -years OS rate was only $25 \%$ in DBC1-positive cases, and was $64 \%$ in DBC1negative cases. In mucinous carcinomas, DBC1 expression was very low compared with other subtypes of ovarian carcinomas (15\% in mucinous carcinoma, $72 \%$ in serous carcinoma, and $100 \%$ in endometrioid carcinoma). However, despite the low frequency of DBC1-positivity and the small number of cases of mucinous carcinoma, DBC1 expression showed borderline significance in OS analysis (Log-rank, $P=0.060$ ). The 10-years OS rates of DBC1negative and $\mathrm{DBC} 1$-positive mucinous carcinomas were $76 \%$ and $33 \%$, respectively. In line with our results, DBC1 expression was associated with a higher nuclear grade of breast carcinoma [39]. Therefore, although the expression rate of $\mathrm{DBC} 1$ differs according to the histologic types, our result suggests that $\mathrm{DBC} 1$ expression might be involved in the progression of ovarian carcinomas, regardless of histologic types. However, further study with a larger group of ovarian carcinoma is needed to clarify the role of $\mathrm{DBC} 1$ in ovarian carcinomas.

\section{Conclusions}

In conclusion, to our knowledge, this is the first study to show that $\mathrm{DBC1}$ is commonly expressed in ovarian carcinomas and its expression is predictive of prognosis of ovarian carcinoma patients, especially in high-grade serous carcinomas and possibly in mucinous carcinomas. In addition, DBC1 expression was significantly associated with BRCA1 expression and their expressions were related with resistance to platinum-based chemotherapy and poor prognosis of ovarian carcinoma patients. Therefore, these results indicate that the expression of DBC1 and BRCA1 might be used for the prediction of prognosis of ovarian carcinomas. Especially, DBC1 expression might be helpful for the prediction of the prognosis of high-grade serous carcinomas. In addition, these findings suggest that $\mathrm{DBC} 1$ and BRCA1could be potential therapeutic targets for the treatment of ovarian carcinomas according to the expression status of DBC1 and BRCA1. However, because of the limited number of non-serous cases of ovarian carcinoma subtypes in this study, further study with more cases is needed to clarify the roles of DBC1 and BRCA1 in various histologic subtypes of ovarian carcinomas.

\section{Abbreviations \\ AR: Androgen receptor; CCAR2: Cell cycle and apoptosis regulator 2; $\mathrm{Cl}$ : Confidence interval; DBC1: Deleted in breast cancer 1; EMT: Epithelial- mesenchymal transition; HR: Hazard ration; LN: Lymph node; OS: Overall survival; RFS: Relapse-free survival; TMA: Tissue microarray.}

\section{Competing interests}

The authors declare that they have no competing interests.

\section{Authors' contributions}

DHJ, HSP, SHP, KMK, MJC, WSM, MJK, and KYJ participated in the study design. DHJ and SHP did the immunohistochemical staining. DHJ, SHP, KMK, MJC, WSM, MJK, and KYJ were involved in data collection and data interpretation. DHJ, HSP, KMK, and KYJ participated in the statistical analysis. DHJ, HSP, SHP, KMK, MJC, WSM, MJK, and KYJ wrote the manuscript. All authors read and approved the final manuscript.

\section{Acknowledgements}

This paper was supported by Research Funds of Chonbuk National University in and by the National Research Foundation of Korea (NRF) grant funded by the Korea government (MSIP) (No. 2008-0062279). We thank DB Leveson-Gower who provided medical writing services.

\section{Author details}

'Department of Obstetrics and Gynecology, Chonbuk National University Medical School and Research Institute of Clinical Medicine, Jeonju, Republic of Korea. ${ }^{2}$ Departments of Pathology, Chonbuk National University Medical School, Research Institute of Clinical Medicine and Research Institute for Endocrine Sciences, Jeonju, Republic of Korea. ${ }^{3}$ Division of Gynecologic Oncology, Department of Obstetrics and Gynecology, Stanford University School of Medicine, Stanford, California, USA.

Received: 29 November 2014 Accepted: 31 January 2015

Published online: 15 February 2015

\section{References}

1. Hamaguchi M, Meth $J$, von Klitzing C, Wei W, Esposito D, Rodgers L, et al. $\mathrm{DBC2}$, a candidate for a tumor suppressor gene involved in breast cancer. Proc Natl Acad Sci U S A. 2002;99:13647-52.

2. Cerami E, Gao J, Dogrusoz U, Gross BE, Sumer SO, Aksoy BA, et al. The cBio cancer genomics portal: an open platform for exploring multidimensional cancer genomics data. Canc Discov. 2012;2:401-4.

3. Gao J, Aksoy BA, Dogrusoz U, Dresdner G, Gross B, Sumer SO, et al. Integrative analysis of complex cancer genomics and clinical profiles using the cBioPortal. Sci Signal. 2013;6:11. 
4. Zhao W, Kruse JP, Tang Y, Jung SY, Qin J, Gu W. Negative regulation of the deacetylase SIRT1 by DBC1. Nature. 2008;451:587-90.

5. Kim JE, Chen J, Lou Z. DBC1 is a negative regulator of SIRT1. Nature. 2008:451:583-6.

6. Sung JY, Kim R, Kim JE, Lee J. Balance between SIRT1 and DBC1 expression is lost in breast cancer. Cancer Sci. 2010;101:1738-44.

7. Kim W, Kim J-E. Deleted in breast cancer 1 (DBC1) deficiency results in apoptosis of breast cancer cells through impaired responses to UV-induced DNA damage. Cancer Lett. 2013;333:180-6.

8. Li Z, Chen L, Kabra N, Wang C, Fang J, Chen J. Inhibition of SUV39H1 methyltransferase activity by DBC1. J Biol Chem. 2009;284:10361-6.

9. Zhang $Y$, Gu Y, Sha S, Kong $X$, Zhu H, Xu B, et al. DBC1 is over-expressed and associated with poor prognosis in colorectal cancer. Int J Clin Oncol. 2014;19:106-12.

10. Bae JS, Park SH, Kim KM, Kwon KS, Kim CY, Lee HK, et al. CK2alpha phosphorylates DBC1 and is involved in the progression of gastric carcinoma and predicts poor survival of gastric carcinoma patients. Int J Cancer. 2015;136:797-809

11. Park HS, Bae JS, Noh SJ, Kim KM, Lee H, Moon WS, et al. Expression of DBC1 and androgen receptor predict poor prognosis in diffuse large B cell lymphoma. Transl Oncol. 2013;6:370-81.

12. Noh SJ, Kang MJ, Kim KM, Bae JS, Park HS, Moon WS, et al. Acetylation status of P53 and the expression of DBC1, SIRT1, and androgen receptor are associated with survival in clear cell renal cell carcinoma patients. Pathology. 2013:45:574-80.

13. Kim JR, Moon YJ, Kwon KS, Bae JS, Wagle S, Yu TK, et al. Expression of SIRT1 and DBC1 is associated with poor prognosis of soft tissue sarcomas. PLoS One. 2013;8:e74738.

14. Lee H, Kim KR, Noh SJ, Park HS, Kwon KS, Park BH, et al. Expression of DBC1 and SIRT1 is associated with poor prognosis for breast carcinoma. Hum Pathol. 2011:42:204-13.

15. Cha EJ, Noh SJ, Kwon KS, Kim CY, Park BH, Park HS, et al. Expression of DBC1 and SIRT1 is associated with poor prognosis of gastric carcinoma. Clin Cancer Res. 2009:15:4453-9.

16. Jang KY, Hwang SH, Kwon KS, Kim KR, Choi HN, Lee NR, et al. SIRT1 expression is associated with poor prognosis of diffuse large B-cell lymphoma. Am J Surg Pathol. 2008;32:1523-31.

17. Park SH, Riley PT, Frisch SM. Regulation of anoikis by deleted in breast cancer-1 (DBC1) through NF-kappaB. Apoptosis. 2013;18:949-62.

18. Joshi P, Quach OL, Giguere SS, Cristea IM. A Functional Proteomics Perspective of DBC1 as a Regulator of Transcription. J Proteomics Bioinform. 2013:Suppl 2:1-17.

19. Chini EN, Chini CC, Nin V, Escande C. Deleted in breast cancer-1 (DBC-1) in the interface between metabolism, aging and cancer. Biosci Rep. 2013;33:e00058

20. Kim SH, Kim JH, Yu EJ, Lee KW, Park CK. The overexpression of DBC1 in esophageal squamous cell carcinoma correlates with poor prognosis. Histol Histopathol. 2012;27:49-58

21. Fu J, Jiang J, Li J, Wang S, Shi G, Feng Q, et al. Deleted in breast cancer 1, a novel androgen receptor (AR) coactivator that promotes AR DNA-binding activity. J Biol Chem. 2009;284:6832-40.

22. Hiraike H, Wada-Hiraike O, Nakagawa S, Koyama S, Miyamoto Y, Sone K, et al. Identification of DBC1 as a transcriptional repressor for BRCA1. Br J Cancer. 2010;102:1061-7.

23. Wiedemeyer WR, Beach JA, Karlan BY. Reversing platinum resistance in high-grade serous ovarian carcinoma: targeting BRCA and the homologous recombination system. Front Oncol. 2014:4:34.

24. Bolton KL, Chenevix-Trench G, Goh C, Sadetzki S, Ramus SJ, Karlan BY, et al. Association between BRCA1 and BRCA2 mutations and survival in women with invasive epithelial ovarian cancer. JAMA. 2012;307:382-9.

25. Therasse P, Arbuck SG, Eisenhauer EA, Wanders J, Kaplan RS, Rubinstein L, et al. New guidelines to evaluate the response to treatment in solid tumors. European Organization for Research and Treatment of Cancer, National Cancer Institute of the United States, National Cancer Institute of Canada. J Natl Cancer Inst. 2000;92:205-16.

26. Kurman RJ, International Agency for Research on Cancer., World Health Organization. WHO classification of tumours of female reproductive organs. 4th ed. Lyon: International Agency for Research on Cancer; 2014.

27. Edge S, Cancer AJCo. AJCC cancer staging handbook: From the AJCC cancer staging manual. New York: Springer; 2010.
28. Allred D, Harvey JM, Berardo M, Clark GM. Prognostic and predictive factors in breast cancer by immunohistochemical analysis. Mod Pathol. 1998;11:155-68.

29. Noh SJ, Bae JS, Jamiyandorj U, Park HS, Kwon KS, Jung SH, et al. Expression of nerve growth factor and heme oxygenase-1 predict poor survival of breast carcinoma patients. BMC Cancer. 2013:13:516.

30. Garg K, Levine DA, Olvera N, Dao F, Bisogna M, Secord AA, et al. BRCA1 immunohistochemistry in a molecularly characterized cohort of ovarian high-grade serous carcinomas. Am J Surg Pathol. 2013;37:138-46.

31. Mylona E, Melissaris S, Nomikos A, Theohari I, Giannopoulou I, Tzelepis K, et al. Effect of BRCA1 immunohistochemical localizations on prognosis of patients with sporadic breast carcinomas. Pathol Res Pract. 2014;210:533-40.

32. Escande C, Chini CC, Nin V, Dykhouse KM, Novak CM, Levine J, et al. Deleted in breast cancer-1 regulates SIRT1 activity and contributes to high-fat diet-induced liver steatosis in mice. J Clin Invest. 2010;120:545-58.

33. Jang KY, Noh SJ, Lehwald N, Tao GZ, Bellovin DI, Park HS, et al. SIRT1 and c-Myc promote liver tumor cell survival and predict poor survival of human hepatocellular carcinomas. PLoS One. 2012;7:e45119.

34. Menssen A, Hydbring P, Kapelle K, Vervoorts J, Diebold J, Luscher B, et al. The c-MYC oncoprotein, the NAMPT enzyme, the SIRT1-inhibitor DBC1, and the SIRT1 deacetylase form a positive feedback loop. Proc Natl Acad Sci U S A. 2012;109:E187-96.

35. Yuan J, Minter-Dykhouse K, Lou Z. A c-Myc-SIRT1 feedback loop regulates cell growth and transformation. J Cell Biol. 2009:185:203-11.

36. Noguchi A, Kikuchi K, Zheng H, Takahashi H, Miyagi Y, Aoki I, et al. SIRT1 expression is associated with a poor prognosis, whereas DBC1 is associated with favorable outcomes in gastric cancer. Cancer Med. 2014;3:1553-61.

37. Bae HJ, Chang YG, Noh JH, Kim JK, Eun JW, Jung KH, et al. DBC1 does not function as a negative regulator of SIRT1 in liver cancer. Oncol Lett. 2012:4:873-7.

38. Jang KY, Kim KS, Hwang SH, Kwon KS, Kim KR, Park HS, et al. Expression and prognostic significance of SIRT1 in ovarian epithelial tumours. Pathology. 2009:41:366-71.

39. Hiraike H, Wada-Hiraike O, Nakagawa S, Saji S, Maeda D, Miyamoto Y, et al. Expression of DBC1 is associated with nuclear grade and HER2 expression in breast cancer. Exp Ther Med. 2011;2:1105-9.

40. Cass I, Baldwin RL, Varkey T, Moslehi R, Narod SA, Karlan BY. Improved survival in women with BRCA-associated ovarian carcinoma. Cancer. 2003;97:2187-95.

41. Chetrit A, Hirsh-Yechezkel G, Ben-David Y, Lubin F, Friedman E, Sadetzki S. Effect of BRCA1/2 mutations on long-term survival of patients with invasive ovarian cancer: the national Israeli study of ovarian cancer. J Clin Oncol. 2008;26:20-5.

42. Nicum S, Roberts C, Boyle L, Kopijasz S, Gourley C, Hall M, et al. A phase II clinical trial of 6-mercaptopurine (6MP) and methotrexate in patients with BRCA defective tumours: a study protocol. BMC Cancer. 2014:14:983.

43. Tutt A, Robson M, Garber JE, Domchek SM, Audeh MW, Weitzel JN, et al. Oral poly(ADP-ribose) polymerase inhibitor olaparib in patients with BRCA or BRCA2 mutations and advanced breast cancer: a proof-of-concept trial. Lancet. 2010;376:235-44.

44. Tutt A, Robson M, Garber J, Domchek S, Audeh M, Weitzel J, et al. Phase II trial of the oral PARP inhibitor olaparib in BRCA-deficient advanced breast cancer. J Clin Oncol (Meeting Abstracts). 2009;27, CRA501.

45. Edmondson RJ, Monaghan JM, Davies BR. The human ovarian surface epithelium is an androgen responsive tissue. Br J Cancer. 2002:86:879-85.

46. Elattar A, Warburton KG, Mukhopadhyay A, Freer RM, Shaheen F, Cross P, et al. Androgen receptor expression is a biological marker for androgen sensitivity in high grade serous epithelial ovarian cancer. Gynecol Oncol. 2012;124:142-7. 